OPEN ACCESS

Edited by:

Kezhen Qi

Shenyang Normal University, China

Reviewed by:

Yongsheng Yang,

Shenyang Normal University, China

Qingrui Zhang,

Yanshan University, China

*Correspondence:

Quanrun Liu

qrliu@163.com

Chuanxiang Zhang

zcx223@hpu.edu.cn

Specialty section

This article was submitted to Nanoscience,

a section of the journal

Frontiers in Chemistry

Received: 10 January 2020 Accepted: 10 February 2020 Published: 28 February 2020

Citation:

Jia J, Sun Y, Zhang Y, Liu Q, Cao J, Huang G, Xing B, Zhang C, Zhang $L$ and Cao Y (2020) Facile and Efficient

Fabrication of Bandgap Tunable Carbon Quantum Dots Derived From Anthracite and Their

Photoluminescence Properties. Front. Chem. 8:123.

doi: 10.3389/fchem.2020.00123

\section{Facile and Efficient Fabrication of Bandgap Tunable Carbon Quantum Dots Derived From Anthracite and Their Photoluminescence Properties}

\author{
Jianbo Jia ${ }^{1,2}$, Yue Sun ${ }^{1}$, Yaojie Zhang ${ }^{1}$, Quanrun Liu ${ }^{1 *}$, Jianliang Cao ${ }^{1}$, Guangxu Huang ${ }^{1,2}$, \\ Baolin Xing ${ }^{1,2}$, Chuanxiang Zhang ${ }^{1,2 *}$, Lina Zhang ${ }^{1}$ and Yijun $\mathrm{Cao}^{2}$ \\ ${ }^{1}$ Henan Key Laboratory of Coal Green Conversion, College of Chemistry and Chemical Engineering, Henan Polytechnic \\ University, Jiaozuo, China, ${ }^{2}$ Henan Province Industrial Technology Research Institute of Resources and Materials, Zhengzhou \\ University, Zhengzhou, China
}

Low-cost and earth-abundant coal has been considered to have a unique structural superiority as carbon sources of carbon quantum dots (CQDs). However, it is still difficult to obtain CQDs from raw coal due to its compactibility and lower reactivity, and the majority of the current coal-based CQDs usually emit green or blue fluorescence. Herein, a facile two-step oxidation approach $\left(\mathrm{K}_{2} \mathrm{FeO}_{4}\right.$ pre-oxidation and $\mathrm{H}_{2} \mathrm{O}_{2}$ oxidation) was proposed to fabricate bandgap tunable CQDs from anthracite. The $\mathrm{K}_{2} \mathrm{FeO}_{4}$ pre-oxidation can not only weaken the non-bonding forces among coal molecules which cause the expansion of coal particles, but also form a large number of active sites on the surface of coal particles. The above effects make the bandgap tunable CQDs (blue, green, or yellow fluorescence) can be quickly obtained from anthracite within $1 \mathrm{~h}$ in the following $\mathrm{H}_{2} \mathrm{O}_{2}$ oxidation by simply adjusting the concentration of $\mathrm{H}_{2} \mathrm{O}_{2}$. All the as-prepared CQDs contain more than 30 at\% oxygen, and the average diameters of which are $<10 \mathrm{~nm}$. The results also indicate that the high oxygen content only can create new energy states inside the band gap of CQDs with average diameter more than $3.2 \pm 0.9 \mathrm{~nm}$, which make the as-prepared CQDs emit green or yellow fluorescence.

Keywords: carbon quantum dots, coal, photoluminescent, anthracite, bandgap engineering

\section{INTRODUCTION}

Carbon quantum dots (CQDs), new zero-dimensional carbon nanomaterials whose size are similar with conventional semiconductor quantum dots but the skeleton is based on carbon, have attracted tremendous research interest after been found (Jaleel and Pramod, 2018; Kaur et al., 2018; Riyanto et al., 2019; Wang et al., 2019; Zhou et al., 2019). And CQDs have been expected to have large potential application in biomedicine (Jaleel and Pramod, 2018), photovoltaic device (Li X. et al., 2015; Kaur et al., 2018), ion detection (Wu et al., 2014; Arumugam and Kim, 2018; Li et al., 2018; Wang et al., 2018; Zhang et al., 2018; Omer et al., 2019), photocatalysis (Yu et al., 2014; Azimirad et al., 2017; Zhang B. et al., 2017; Syed et al., 2019), and other fields due to their fascination optical and electro-optical properties (Shao et al., 2016; Pramanik et al., 2018).

Coal is consist of angstrom-sized or nanometer-sized crystalline carbon linked by amorphous carbon and polymerized aromatic hydrocarbon (Thiyagarajan et al., 2016). These crystalline carbon 
domains are abundant in coal and the size of them meet requirements of CQDs (Dong et al., 2014; Hoang et al., 2018). Additionally, the cheapest price and substantial deposits of coal, in contrast to crystalline carbon such as graphene, carbon tubes and fullerenes, have attracted tremendous interest and efforts in developing preparation methods of CQDs from coal. Up to now, CQDs have been successfully prepared from coal by different methods (Ye et al., 2013; Dong et al., 2014; Hu et al., 2015, 2016; Li M. et al., 2015; Sasikala et al., 2016; Li et al., 2017; Liu X. et al., 2018; Saikia et al., 2019). Ye et al. (2013) employed concentrated sulfuric acid and nitric acid to exfoliate CQDs from coal at $100^{\circ}$ or $120^{\circ} \mathrm{C}$ for $24 \mathrm{~h}$. Similarly, CQDs were obtained through refluxing coal in $5 \mathrm{M} \mathrm{HNO}_{3}$ at $120^{\circ} \mathrm{C}$ for $12 \mathrm{~h}$ (Dong et al., 2014). However, there are some drawbacks to the above methods, such as the longer reaction time and the inherent difficulty in separation of CQDs from the mixture which contains a large amount of inorganic salts that formed during the neutralization phase via the addition of bases. Hence, in order to optimizing the preparation conditions of CQD from coal, selective depolymerization of coal in an oxidizing supercritical fluid was proposed by Sasikala et al. (2016). They isolated CQDs in supercritical water under the conditions of $400^{\circ} \mathrm{C}$ and 25 $\mathrm{MPa}$ within $2 \mathrm{~h}$. Although this way could observably shorten the time to prepare CQDs, the unattainable reaction conditions hampered the large scale preparation of CQDs. Whereupon, greener oxidants $\left(\mathrm{H}_{2} \mathrm{O}_{2}, \mathrm{O}_{3}\right)$ were utilized to produce CQDs from coal under milder reaction conditions (Hu et al., 2016; Liu X. et al., 2018).

Despite these efforts, most current CQDs prepared from coal show green or blue fluorescent. And the difficulties in obtaining a defined and desired bandgap have largely hindered the applications of CQDs for a particular purpose (Yan et al., 2018). Hence, in order to obtain narrow bandgap CQDs (yellow to red fluorescent) from coal, there have been more efforts to tailor bandgap of CQDs. The bandgap of CQDs opening is due to the quantum confinement effect (Pan et al., 2010). Consequently, tuning the lateral size of CQD is one of the common strategies for narrowing bandgap of CQD. Different nanometer-sized CQDs were prepared from various coal or coke which possess different-sized graphene domains by strong acidic oxidation (Ye et al., 2013; Hu et al., 2015). In addition, different nanometer-sized CQDs can also be prepared solely from anthracite in concentrated $\mathrm{H}_{2} \mathrm{SO}_{4}$ and $\mathrm{HNO}_{3}$, and the size control of CQDs was achieved through cross-flow ultrafiltration, controlling the reaction temperature of the oxidation process or conjugating pristine CQDs with poly aromatic rings (Ye et al., 2015; Yan et al., 2018). Another approach for narrowing the bandgap is forming intramolecular Z-schemes structure via functionalization of pristine CQDs with electron-donating chemical groups (Yan et al., 2018). Much progress has been made, but many more problems need solving before CQDs with varying bandgaps can be feasibly produced from coal in large-scale, such as long production phases, critical synthesis conditions or expensive reagents. Therefore, a simple, fast and facile synthesis method is still highly desirable.

Here we report a two-step, facile and fast method to fabricate CQDs with varying bandgaps solely from anthracite. In the first step, which is called pre-oxidation stage, anthracite was oxidized with potassium ferrate $\mathrm{K}_{2} \mathrm{Fe}^{\mathrm{VI}} \mathrm{O}_{4}$ as an oxidant in $\mathrm{H}_{2} \mathrm{SO}_{4}$ medium to obtain the oxidized coal. The $\mathrm{sp}^{3}$-hybridized carbon atoms can be selectively oxidized and abundant oxygen-containing groups were produced after $\mathrm{K}_{2} \mathrm{FeO}_{4} / \mathrm{H}_{2} \mathrm{SO}_{4}$ treatment (Zhang and $\mathrm{Xu}, 2015$ ). This can substantially improve the chemical reaction activity and wet ability of anthracite. In the second step, CQDs with varying bandgaps can be fast fabricated from the oxidative coal using $\mathrm{H}_{2} \mathrm{O}_{2}$ as an oxidant by simply controlling the concentration of $\mathrm{H}_{2} \mathrm{O}_{2}$.

\section{EXPERIMENTAL}

\section{Materials}

Anthracite collected from Taixi (Inner Mongolia Province, China) without pretreatment was crushed and ground to powder (about 200 mesh). $\mathrm{H}_{2} \mathrm{O}_{2}\left(30\right.$ wt.\%) and $\mathrm{H}_{2} \mathrm{SO}_{4}$ (98\%) were purchased from Sinopharm Chemical Reagent Co., Ltd., China. $\mathrm{K}_{2} \mathrm{FeO}_{4}$ was purchased from Shanghai Mecoxlane International MailorderCo., Ltd., China. Polyethersulfone filter membranes $(0.22 \mu \mathrm{m})$ were purchased from Jinteng Experimental Equipment Co., Ltd., China. All reagents were used as received unless otherwise noted. Deionized water was used for all experiments.

\section{Pre-oxidation of Anthracite}

Two gram anthracite and $100 \mathrm{ml}$ of concentrated $\mathrm{H}_{2} \mathrm{SO}_{4}$ were mixed in a $250 \mathrm{ml}$ flask. Then $2.5 \mathrm{~g}$ potassium ferrate was slowly added in small doses to avoid overheating. The reaction mixture was kept at $40^{\circ} \mathrm{C}$ for $1 \mathrm{~h}$ under magnetic stirring. Once the reaction had finished, the mixture was centrifuged to recycle the concentrated $\mathrm{H}_{2} \mathrm{SO}_{4}$. The precipitate was poured in to $100 \mathrm{ml}$ water and stand for $30 \mathrm{~min}$. The oxidized coal was obtained by repeated centrifugation and washing with water until the $\mathrm{pH}$ of the supernatant approached 7.

\section{Synthesis of CQDs}

One gram oxidized coal was mixed with $50 \mathrm{ml} \mathrm{H}_{2} \mathrm{O}_{2}$ solution, then the obtained mixture was stirred and reacted at $100^{\circ} \mathrm{C}$ for $1 \mathrm{~h}$. Subsequently, the unreacted coal was removed via centrifugation at $8,000 \mathrm{rpm}$ for $5 \mathrm{~min}$. The supernatant was then filtered through a $0.22 \mu \mathrm{m}$ filter membrane to remove the larger fragments and the filtrate was dialyzed in 1,000 Da dialysis bag. After purification, the filtrate was freeze-dried to obtain solid CQDs. In order to adjust the bandgap of CQDs, only the concentration of $\mathrm{H}_{2} \mathrm{O}_{2}$ was changed from 30 to $10 \%$, and the other experimental conditions remain unchanged.

\section{Characterization}

Transmission electron microscopy (TEM) images were conducted with a Tecnai G2 F20 instrument (FEI, USA) operated at $200 \mathrm{kV}$. The scanning electron microscope (SEM) was performed on a Quanta FEG 250 field-emission SEM system (FEI, USA). The fluorescence spectra were measured by a Cary Eclipse spectrophotometer (Varian, USA). Xray photoelectron spectroscopy (XPS) measurement was performed on an ESCALAB250 Xi photoelectron spectrometer 
(Thermo Fisher Scientific, USA). The Fourier-transform infrared spectroscopy (FT-IR) and Ultraviolet-visible (UV-Vis) absorption spectroscopy were obtained from a VERTEX 70 FTIR spectrometer (Bruker, Germany) and a Pgeneral TU1810 spectrometer (Pgeneral, China), respectively. Raman spectra were recorded using a microscopic confocal raman spectrometer (Renishaw, UK) with an argon ion laser $(\lambda=$ $514 \mathrm{~nm}$ ) at ambient temperature. The X-ray diffraction (XRD) patterns of all samples were recorded on a Bruker D8 Advance (Bruker, Germany) with a $\mathrm{Cu} \mathrm{K} \alpha \mathrm{X}$-ray radiation source
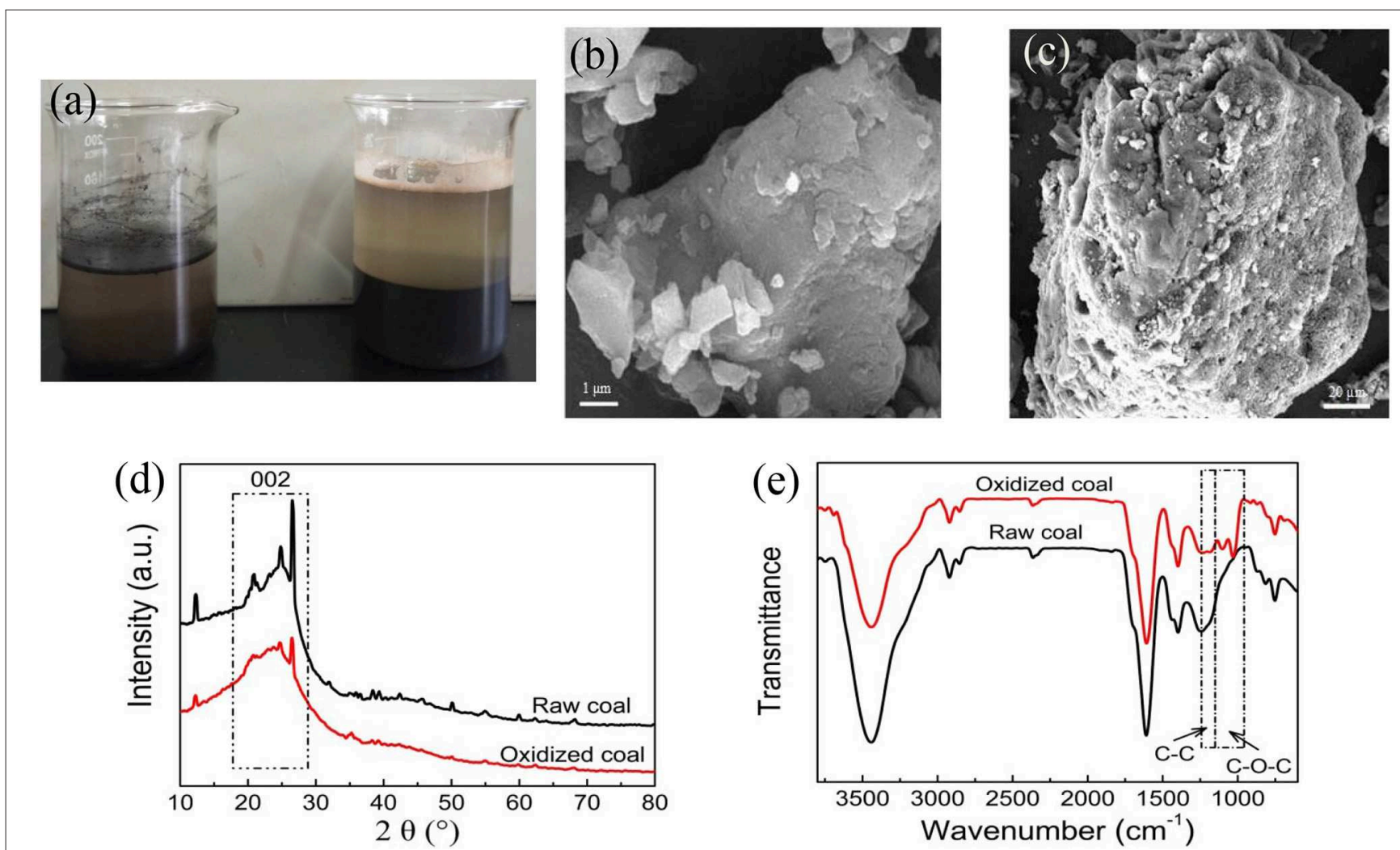

(f)
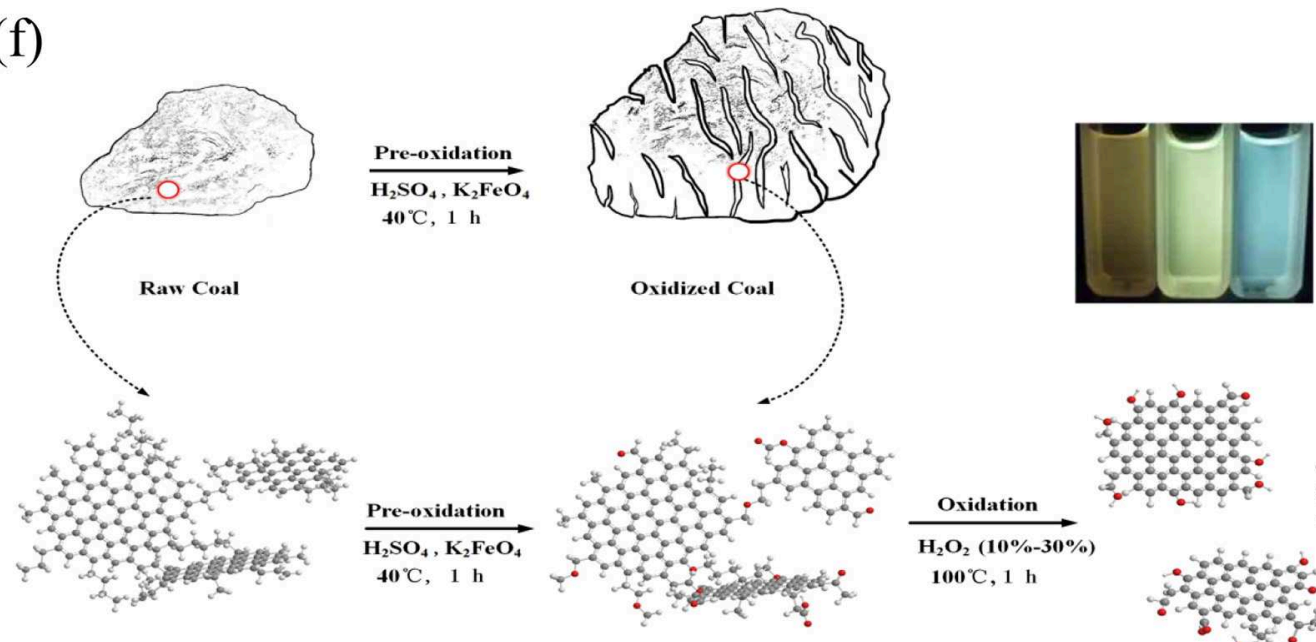

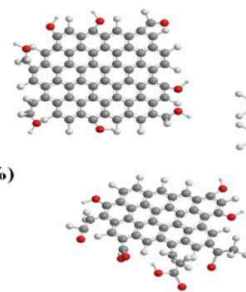

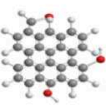

\section{$\bigcirc \mathrm{C} \bigcirc \mathrm{H} \quad \bigcirc \quad \mathrm{O}$}

FIGURE 1 | (a) The disperse of anthracite in water (left: raw coal, right: oxidized coal). SEM images of (b) raw coal and (c) oxidized coal. (d) XRD and (e) FTIR spectra of raw coal and oxidized coal. (f) Schematic illustration of CQDs synthesis. 
$(\lambda=0.15418 \mathrm{~nm})$, and the scattering angles $(2 \theta)$ range from $10^{\circ}$ to $80^{\circ}$.

\section{RESULTS AND DISCUSSION \\ Characterization of Oxidized Coal and Preparation Principle Analysis of Bandgap Tunable CQDs}

Raw anthracite was oxidized with $\mathrm{K}_{2} \mathrm{FeO}_{4} / \mathrm{H}_{2} \mathrm{SO}_{4}$ to improve its surface reactivity. As shown in Figure 1a, the wet ability of pulverized coal is very poor, and most of which floated on the surface of water before oxidized. However, pulverized coal could be very well-moistened with water and the volume of raw coal expanded significantly after the pre-oxidation, indicating that the pre-oxidation has the powerful influence on the structure and properties of anthracite. SEM was used to observe the microscopic morphology change of anthracite before and after the pre-oxidation (Figures $\mathbf{1 b}, \mathbf{c}$ ). Compared with the compact structure of raw coal, there are lots of crevices or pores on the surface of the oxidized coal, which may be offer much more

A
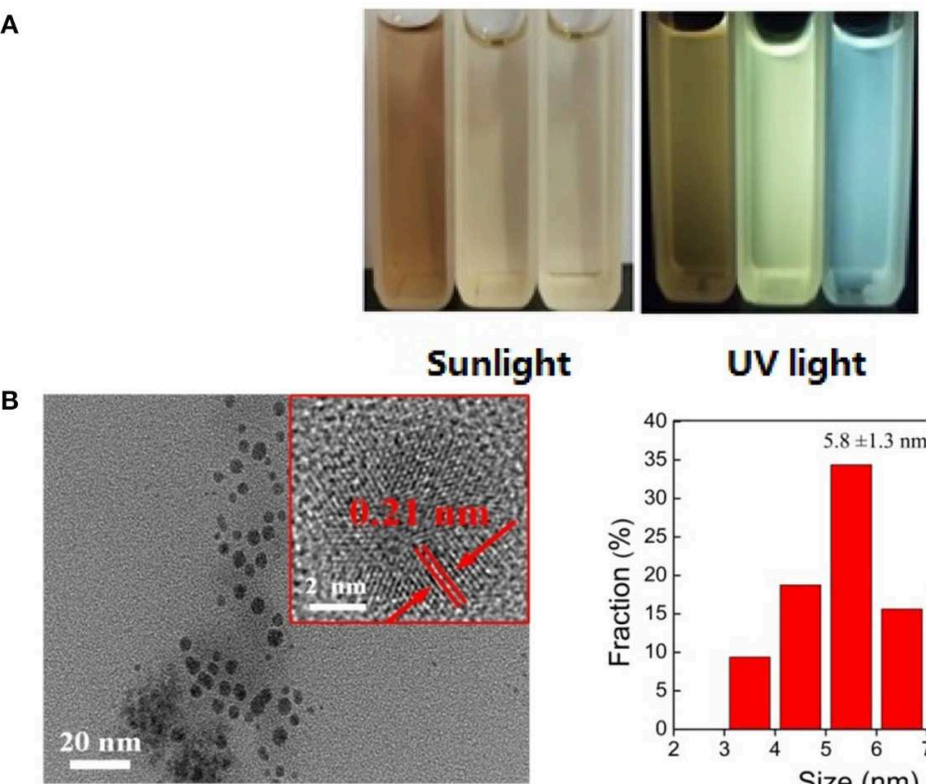

UV light

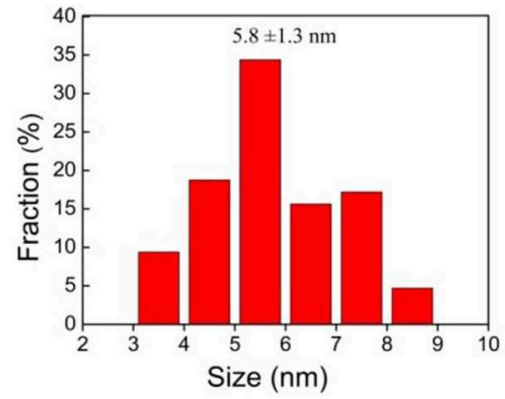

C
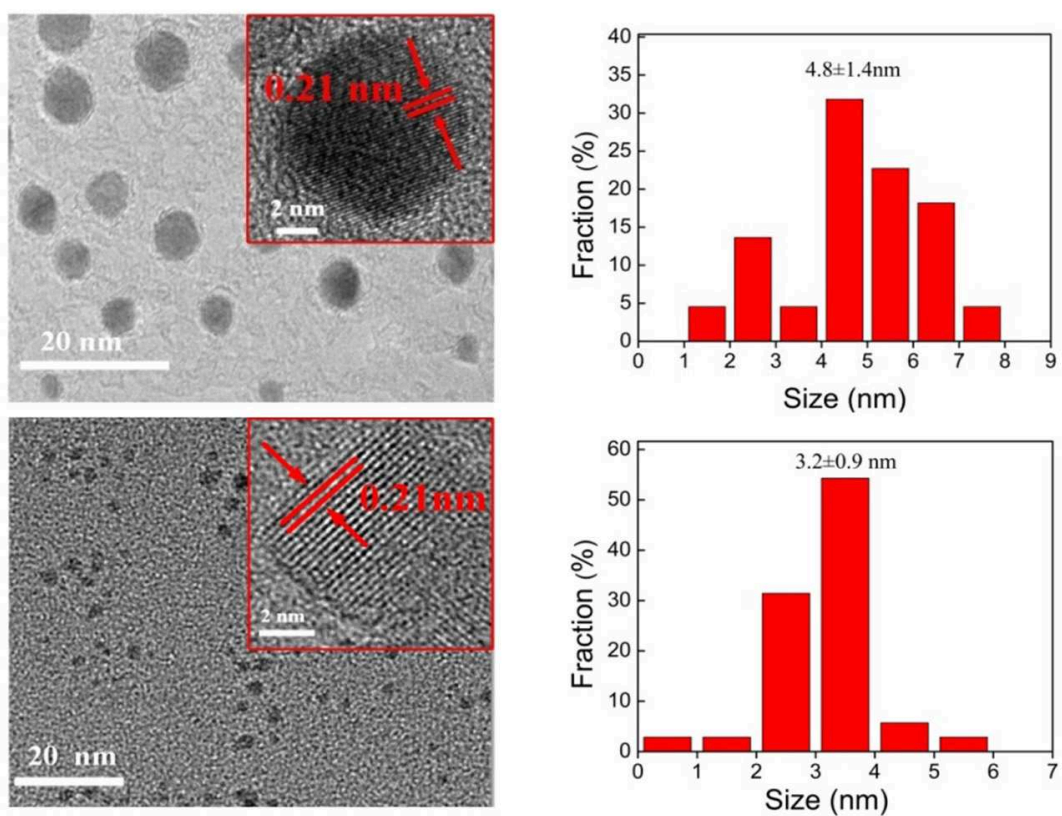

FIGURE 2 | (A) Aqoeous solution of CQDs under sunlight and UV light (the left cuvette is CQDs-N10-Y solution, the middle cuvette is CQDs-N20-G solution and the right cuvette is CQDs-N2O-G solution). The TEM images, HRTEM images, and size distributions of (B) CQDs-N10-Y, (C) CQDs-N20-G, and (D) CQDs-N30-B. 
active sites to react with $\mathrm{H}_{2} \mathrm{O}_{2}$ reactant used subsequently for fabrication of CQDs. To obtain more information about the structure change of anthracite after the pre-oxidation, original and oxidized samples were analyzed by the X-ray diffraction (XRD) and Fourier transform infrared spectroscopy (FTIR). The XRD spectra (Figure 1d) show that one broad peak observed at $24.5^{\circ}$ is attributed to the 002 plane of graphite lattice for both original and oxidized samples (Duraia et al., 2018; Liu Y. et al., 2018). The position and full width at half maximum intensity of 002 peak have little change before and after the pre-oxidation, indicating that the crystalline carbon domains of anthracite were not corrupted in the process of pre-oxidation. The FTIR spectra are shown in Figure 1e. Major structural changes during pre-oxidation for anthracite mainly occurred around 1,000 and $1,300 \mathrm{~cm}^{-1}$ region. The intensity of $1,150-$ $1,250 \mathrm{~cm}^{-1}$ assigned to saturated aliphatic skeletal C-C vibration was found to be weaker and there was a group of peaks in the region $1,150-950 \mathrm{~cm}^{-1}$ due to stretching vibration of $\mathrm{C}$ $\mathrm{O}$ (C-O-C or phenolic) for oxidized coal (Okolo et al., 2015; Xing et al., 2017; Qiu et al., 2020), suggesting that a large number of oxygen-containing functional groups were formed and a part of aliphatic carbon atoms were consumed during the pre-oxidation process.

The above results can be interpreted as the selective oxidation of $\mathrm{K}_{2} \mathrm{FeO}_{4} / \mathrm{H}_{2} \mathrm{SO}_{4}$, namely the $\mathrm{sp}^{3} \mathrm{C}$ - $\mathrm{C}$ bonds were oxidized while the $\mathrm{C}=\mathrm{C}$ bonds remained intact (Zhang and $\mathrm{Xu}, 2015$ ). $\mathrm{H}_{2} \mathrm{SO}_{4}$ and $\mathrm{K}_{2} \mathrm{FeO}_{4}$ can quickly intercalate into the coal molecule interval. The aliphatic portions were oxidized while the oxidant reacts with $\mathrm{H}^{+}$or water to produce a lot of oxygen gas (Peng et al., 2015), these results can break the non-covalent interactions (hydrogen bonds, $\pi-\pi$ interactions, van der Walls interactions and electrostatic interactions) (Niekerk et al., 2010) and cause the significant swelling bulk of powdered coal. Overall, compared to original coal, the oxidized coal possesses higher reactivity in reaction with $\mathrm{H}_{2} \mathrm{O}_{2}$. The entire fabrication process of CQDs requires only $1 \mathrm{~h}$, and the as-prepared CQDs with varying bandgaps were obtained by solely controlling the concentration of $\mathrm{H}_{2} \mathrm{O}_{2}$. The CQDs formation mechanism is shown in Figure 1f.

\section{Characterization of Bandgap Tunable CQDs}

The CQDs synthesized with different concentration of $\mathrm{H}_{2} \mathrm{O}_{2}$ at $100^{\circ} \mathrm{C}$ are denote as CQDs- Nx-y where " $\mathrm{N}$ " signifies "Concentration," " $\mathrm{x}$ " signifies the concentration of $\mathrm{H}_{2} \mathrm{O}_{2}$ and " $y$ " signifies the fluorescent color of CQDs solution under UV lamp $(365 \mathrm{~nm})$ irradiation, such as yellow, green, and blue are represented by $\mathrm{Y}, \mathrm{G}$ and $\mathrm{B}$, respectively. The change of CQDs in bandgap is visualized in Figure 2A, where the as-prepared CQDs solutions emit from blue to yellow under UV lamp (365 nm) irradiation. An of the interesting finding was that the bandgap of CQDs is red-shifted from blue to yellow with decreasing of $\mathrm{H}_{2} \mathrm{O}_{2}$ concentration from 30 to $10 \%$. The production yields of CQDs-N10-Y, CQDs-N20-G, and CQDs-N30-B are 18.9, 12.6, and $4.3 \%$, respectively. The production yield of CQDs is the ratio between the obtained CQDs solid powder and the amount of coal.
The microstructure of the as-prepared CQDs was investigated by transmission electron microscopy (TEM). The TEM images and the size distributions of the as-prepared CQDs are shown

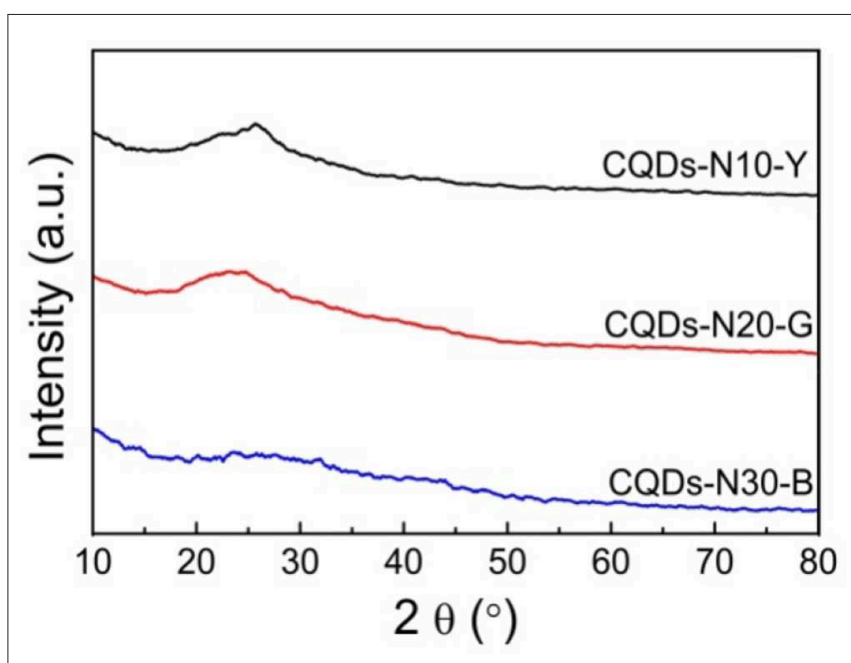

FIGURE 3 | XRD patterns of CQDs-N10-Y, CQDs-N20-G, and CQDs-N30-B.

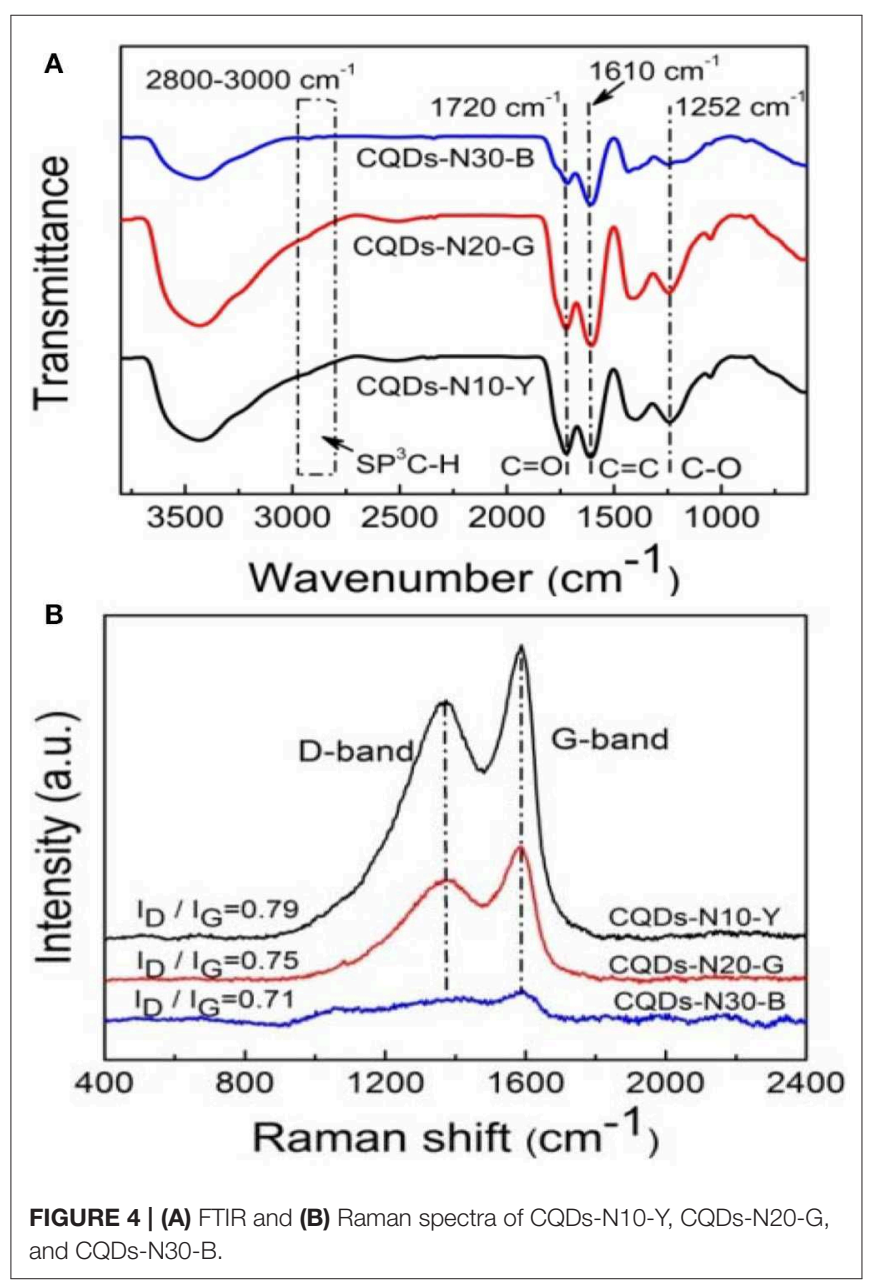


in Figures 2B-D and the high-resolution TEM (HRTEM) of the as-prepared CQDs is inset in the corresponding TEM. It was found that the diameters of the as-prepared CQDs were relatively uniform and all the as-prepared CQDs have a quasi-spherical shape. The HRTEM images reveal that the as-prepared CQDs have highly crystalline structure with a lattice spacing of ca. $0.21 \mathrm{~nm}$, which is corresponding to the (100) diffraction facets of graphite carbon (Tian et al., 2017). The size distributions of CQDs-N10-Y, CQDs-N20-G, and CQDs-N30-B are 5.8 $\pm 1.3,4.8$ \pm 1.4 , and $3.2 \pm 0.9 \mathrm{~nm}$, respectively.

The XRD patterns of the as-prepared CQDs (Figure 3) show that one broad peak (about $25^{\circ}$ ) ascribed to the 002 plane of graphite lattice is observed, and this peak of CQDs-N30-B is almost unseeable due to its smaller size. The CQDs could also be prepared by directly oxidation of anthracite with $\mathrm{H}_{2} \mathrm{O}_{2}$, but the diameters of which are mainly distributed from 1 to $3 \mathrm{~nm}$ significantly smaller than that of the CQDs prepared in this work and they emit blue under UV light (Hu et al., 2016). This indicates the pre-oxidation of anthracite plays a key role in the bandgap adjustment of CQDs which can shorten the oxidative time to avoid the excessive oxidation of crystalline carbon domains of anthracite. In general, as the size of CQDs increases, the bandgap narrows. Therefore, at least initially, this work was designed to tune the bandgap by changing the size of CQDs, however the average diameters of the as-prepared CQDs in this work are all $<10 \mathrm{~nm}$. In the literature (Pan et al., 2010), the CQDs with $9.6 \mathrm{~nm}$ average diameter were fabricated from graphene oxide by hydrothermal treatment at $200^{\circ} \mathrm{C}$, and they emit bright blue under UV light, indicating that the major factor adjusting the bandgap of the as-prepared CQDs isn't the size.
To further understand the effect of reaction conditions on the functional groups, FTIR, Raman and X-ray photoelectron spectroscopy (XPS) and were applied to investigate the structure of CQDs. The FTIR spectra of CQDs are shown in Figure 4A. The absorption peaks at $2,800-3,000 \mathrm{~cm}^{-1}$ are assigned to stretching vibrations of aliphatic $\mathrm{C}-\mathrm{H}$. The strong absorption peak at $1,610 \mathrm{~cm}^{-1}$ are in associated with stretching vibration of aromatic $\mathrm{C}=\mathrm{C}$. Compared to the oxidized coal, absorption peaks $\left(2,800-3,000 \mathrm{~cm}^{-1}\right)$ almost disappear in the FTIR spectra of CQDs, indicating the aliphatic carbon chains which link the crystalline carbon domains of coal have be selectively oxidized into $\mathrm{CO}_{2}$ and $\mathrm{H}_{2} \mathrm{O}$ by $\mathrm{H}_{2} \mathrm{O}_{2}$ ( $\mathrm{Hu}$ et al., 2016). Besides, the peaks at $1,720 \mathrm{~cm}^{-1}$ and $1,252 \mathrm{~cm}^{-1}$ are attributed to the $\mathrm{C}=\mathrm{O}$ stretching, the $\mathrm{O}-\mathrm{H}$ deformation and $\mathrm{C}-\mathrm{O}$ stretching of carboxylic acid, respectively, which make CQDs hydrophilic and stable dispersion in water. The Raman spectra of the as-prepared CQDs (Figure 4B) reveal a D band at $1,367 \mathrm{~cm}^{-1}$ and a $\mathrm{G}$ band at $1,587 \mathrm{~cm}^{-1}$, which correspond to disordered structures and graphitic structures, respectively, of carbon materials (Ding et al., 2015; Huang et al., 2020). The intensity ratio for the D to G bands can be used to reflect the level of disorder in carbon materials

TABLE 1 | Element content of the as-prepared CQDs calculated based on XPS analysis (atom \%).

\begin{tabular}{lcc}
\hline Samples & C & O \\
\hline CQDs-N10-Y & 63.8 & 34.4 \\
CQDs-N2O-G & 57.1 & 40.9 \\
CQDs-N30-B & 55.9 & 42.6
\end{tabular}

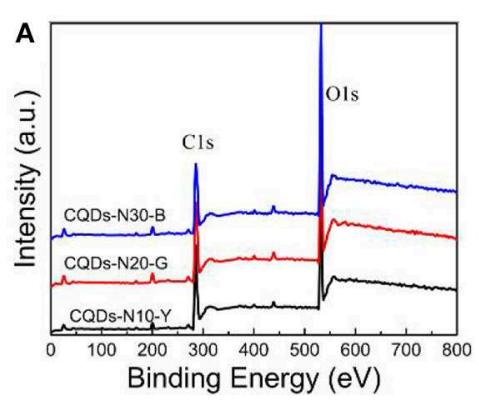

C

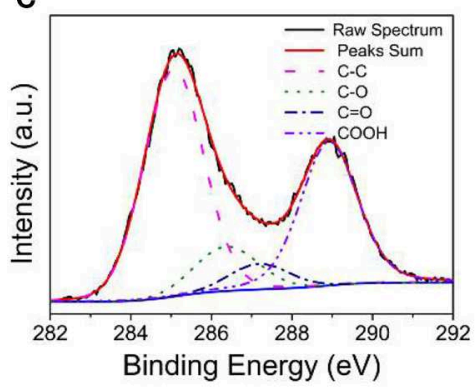

D

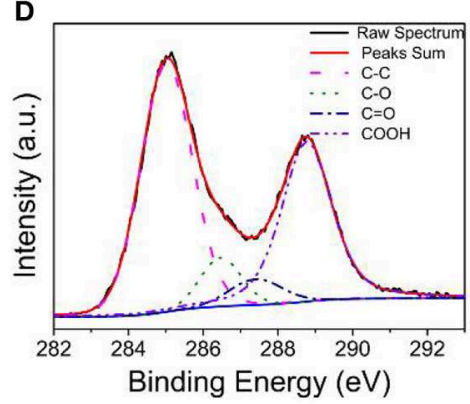

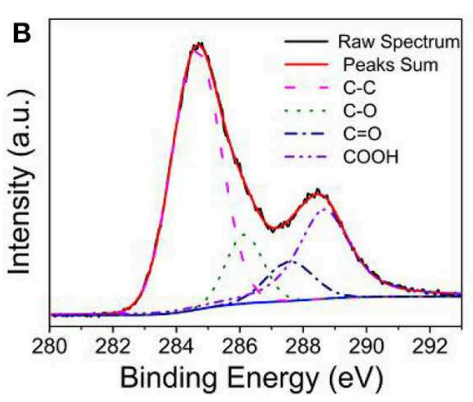

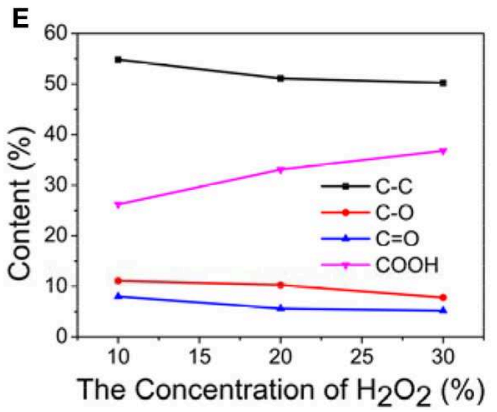

FIGURE 5 | (A) XPS survey spectra of CQDs-N10-Y, CQDs-N20-G, and CQDs-N30-B. C 1s high resolution XPS spectra of (B) CQDs-N10-Y, (C) CQDs-N20-G, and (D) CQDs-N3O-B. (E) Summary of relative percentage of different functional groups on the surface of CQDs from (B) to (D). 
(Ma et al., 2019). The values of $\mathrm{I}_{\mathrm{D}} / \mathrm{I}_{\mathrm{G}}$ for CQDs-N10-Y, CQDsN20-G, and CQDs-N30-B are 0.79, 0.75, and 0.71, respectively, suggesting the as-prepared CDQs possess much more ordered graphite structures with decreasing of size of CQDs.

The chemical structures at the surface of the as-prepared CQDs were further investigated by X-ray photoelectron spectroscopy (XPS) analyses. The XPS survey spectra of CQDs (Figure 5A) show that the as-prepared CQDs primarily consist of carbon and oxygen (Zhang Q. et al., 2017, 2019; Zeng et al., 2020). It can be seen that the oxygen content gradually increases from 34.4 to $42.6 \%$ with the increasing of the $\mathrm{H}_{2} \mathrm{O}_{2}$ concentration in Table 1, indicating the higher the concentration of $\mathrm{H}_{2} \mathrm{O}_{2}$, the stronger the oxidation ability, due to the increase of the number of-OH radical. As shown in Figures 5B-D, the high resolution $\mathrm{C} 1 \mathrm{~s}$ spectra of CQDs can be conceived into four peaks at $285 \mathrm{eV}(\mathrm{C}-\mathrm{C} / \mathrm{C}=\mathrm{C} / \mathrm{C}-\mathrm{H}), 286.3 \mathrm{eV}(\mathrm{C}-\mathrm{O}), 287.4 \mathrm{eV}(\mathrm{C}$ $=\mathrm{O}$ ), and $288.8 \mathrm{eV}(\mathrm{COOH})$ (Roy et al., 2014; Shi et al., 2015; Moonrinta et al., 2018; Pacquiao et al., 2018), suggesting the presence of large quantities of oxygen-containing functional groups, especially carboxyl groups. The relative abundances of these components are summarized in Figure 5E.

\section{Optical Properties of Bandgap Tunable CQDs}

The optical properties of as-prepared CQDs were explored by UV-vis absorption and photoluminescence (PL) spectroscopy. The UV-vis absorption spectra of as-prepared CQDs are shown in Figure 6A. It is clear that there are a strong peak and a weak peak at 221 and $298 \mathrm{~nm}$ corresponding to $\pi-\pi^{*}$ transition of $\mathrm{C}=\mathrm{C}$ and $\mathrm{n}-\pi^{*}$ transition of $\mathrm{C}=\mathrm{O}$, respectively (Dehghani et al., 2018; Yang et al., 2018). Apparently, the shoulder peak at $298 \mathrm{~nm}$ is almost invisible in the spectrum of CQDs-N10-Y, indicating that the surface content of $\mathrm{COOH}$ is relatively lower in CQDsN10-Y (Zhang et al., 2016). This phenomenon is consistent with the results of XPS analysis. The adsorption of CQDs-N30$\mathrm{G}$ occurs mainly in the UV region $(<400 \mathrm{~nm})$. However, the absorption regions of YCQDs-N10-Y and CQDs-N20-G extend to the visible region $(400-650 \mathrm{~nm})$, and the adsorption is more prominent for CQDs-N10-Y, suggesting more narrowing of the bandgap (Choi et al., 2018).

The photoluminescence (PL) spectra of the as-prepared CQDs excited at the different wavelengths are shown in Figures 6B-D. The maximum emission peak shifts from $\sim 525$ to $\sim 450 \mathrm{~nm}$ as the concentration of $\mathrm{H}_{2} \mathrm{O}_{2}$ increases from 10 to $30 \%$. Moreover, it is worth noting that like most CQDs (Zhu et al., 2013; Liu Q. et al., 2018; Chen et al., 2020), the PL behavior of CQDsN30-B exhibits the emission wavelength is dependence of the excitation wavelength and the intensity of PL decreases with increase of excitation wavelength. But the emission peaks lie in almost the same wavelength $(\sim 510$ or $\sim 525 \mathrm{~nm})$ with increasing excitation wavelength for both CQDs-N10-Y and CQDs-N20G. Two distinct emission modes were proposed to interpretive the PL mechanism of CQDs, i.e., intrinsic emission mode and extrinsic emission mode (Liu et al., 2013). The emission peak near $450 \mathrm{~nm}$ when excited near $325 \mathrm{~nm}$ is considered as the intrinsic emission of $\mathrm{sp}^{2}$ carbon hexagons. The emission peak
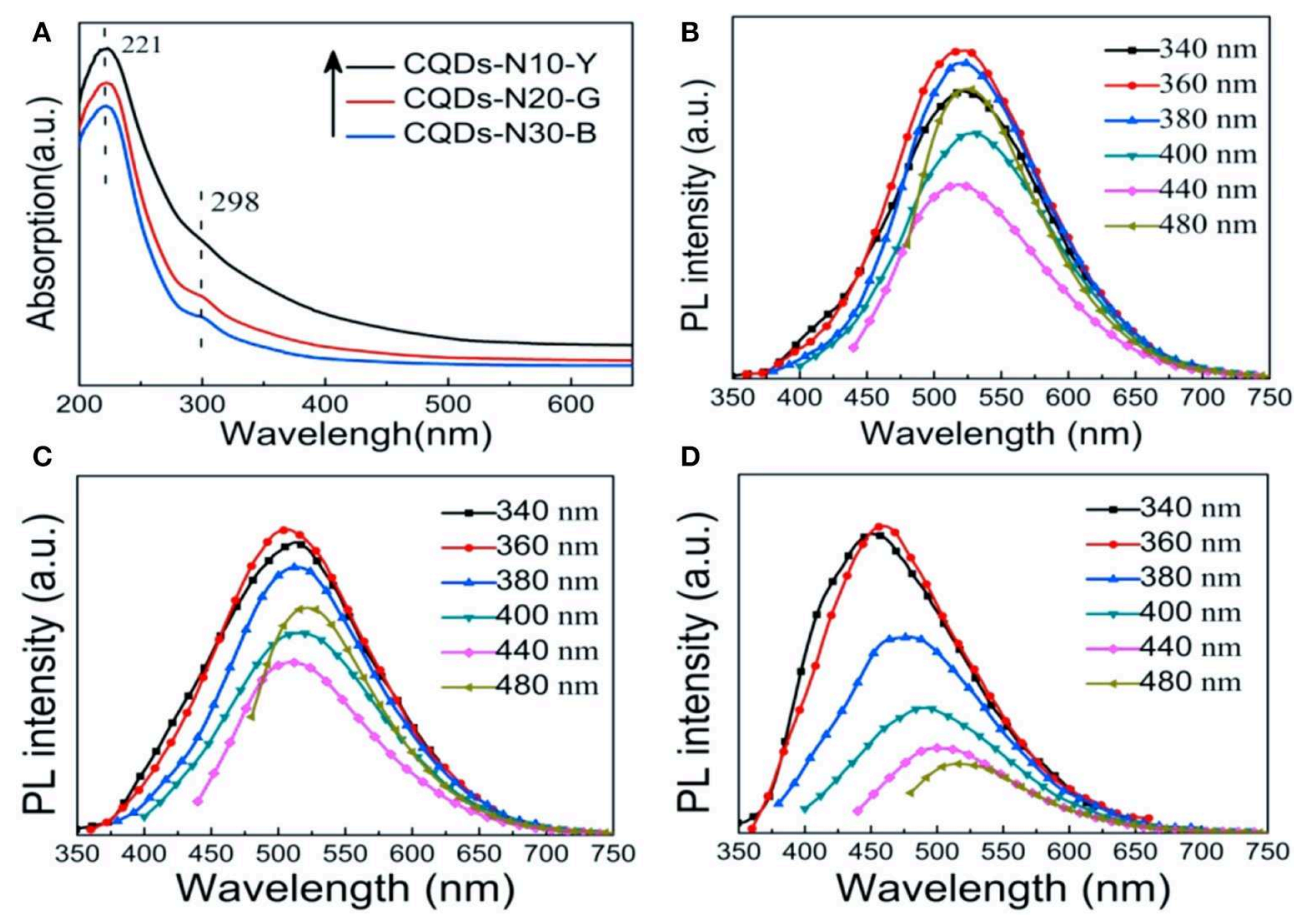

FIGURE 6 | (A) UV-vis absorption spectra of CQDs-N10-Y, CQDs-N20-G, and CQDs-N30-B. Photoluminescence (PL) spectra of (B) CQDs-N10-Y, (C) CQDs-N20-G, and (D) CQDs-N30-B at different excitation wavelengths. 
near $500 \mathrm{~nm}$ when excited with $325 \mathrm{~nm}$ and above is attribute to the extrinsic emission due to the defects of CQDs including oxygen-containing functional groups or $\mathrm{sp}^{3}$ carbon. The work of Yoon et al. (2016) indicated that oxygen-containing functional groups may create new energy states (extrinsic state) inside the band gap of CQDs resulting in the red shift of PL emission. The results of FTIR and XPS analysis show quantities of oxygencontaining functional groups exist on the surfaces of CQDsN10-Y, CQDs-N20-G and CQDs-N10-B, but the amount of $\mathrm{sp}^{3}$ carbon is very low. Therefore, the emission peaks near $520 \mathrm{~nm}$ of CQDs-N10-Y and CQDs-N20-G can be attributed to extrinsic emission mode due to the new energy states created by oxygencontaining functional groups. But it is interesting to note that although the oxygen content of CQDs-N30-B is the highest in all the as-prepared CQDs, the emission peak of which is still near $450 \mathrm{~nm}$ excited near $325 \mathrm{~nm}$. We propose that this is because in the case of smaller size of CQDs-N30-B $(3.2 \pm 0.9 \mathrm{~nm})$ the emission is likely dominated by the intrinsic transitions rather than the extrinsic, oxygen defects related transitions. So, our results suggest that for small CQDs prepared by the method described here both intrinsic and extrinsic transitions contribute to the observed CQDs PL, where the former dominate the PL in CQDs-N30-B and the latter dominate the PL in CQDs-N10-Y and CQDs-N20-G.

\section{CONCLUSIONS}

In conclusion, we have developed a facile, fast, and green method to prepare bandgap tunable CQDs solely from anthracite. The emission color of CQDs can be adjusted from yellow to blue

\section{REFERENCES}

Arumugam, N., and Kim, J. (2018). Synthesis of carbon quantum dots from broccoli and their ability to detect silver ions. Mater. Lett. 219, 37-40. doi: 10.1016/j.matlet.2018.02.043

Azimirad, R., Safa, S., Ebrahimi, M., Yousefzadeh, S., and Moshfegh, A. Z. (2017). Photoelectrochemical activity of graphene quantum dots/hierarchical porous $\mathrm{TiO}_{2}$ photoanode. J. Alloy. Compd. 721, 36-44. doi: 10.1016/j.jallcom.2017.05.301

Chen, Y., Sun, X., Pan, W., Yu, G., and Wang, J. (2020). Fe3+-sensitive carbon dots for detection of $\mathrm{Fe}^{+}$in Aqueous solution and intracellular imaging of $\mathrm{Fe}^{+}$ inside fungal cells. Front. Chem. 7:911. doi: 10.3389/fchem.2019.00911

Choi, J., Kim, N., Oh, J., and Kim, F. S. (2018). Bandgap engineering of nanosized carbon dots through electron-accepting functionalization. J. Ind. Eng. Chem. 65, 104-111. doi: 10.1016/j.jiec.2018.04.018

Dehghani, A., Ardekani, S. M., Hassan, M., and Gomes, V. G. (2018). Collagen derived carbon quantum dots for cell imaging in $3 \mathrm{D}$ scaffolds via two-photon spectroscopy. Carbon 131, 238-245. doi: 10.1016/j.carbon.2018. 02.006

Ding, H., Yu, S., Wei, J., and Xiong, H. (2015). Full-color light-emitting carbon dots with a surface-state-controlled luminescence mechanism. ACS Nano 10, 484-491. doi: 10.1021/acsnano.5b05406

Dong, Y., Lin, J., Chen, Y., Fu, F., Chi, Y., and Chen, G. (2014). Graphene quantum dots, graphene oxide, carbon quantum dots and graphite nanocrystals in coals. Nanoscale 6, 7410-7415. doi: 10.1039/C4NR01482K

Duraia, E. M., Niu, S., Beall, G. W., and Rhodes, C. P. (2018). Humic acid-derived graphene- $\mathrm{SnO}_{2}$ nanocomposites for high capacity lithium-ion battery anodes. J. Mater. Sci. 29, 8456-8464. doi: 10.1007/s10854-018-8858-x under UV light. The bandgap change of as-prepared CQDs can be achieved by simply controlling the concentration of $\mathrm{H}_{2} \mathrm{O}_{2}$. The morphology, size and PL properties of the as-prepared CQDs indicate that the blue luminescence may originate from the intrinsic emission, but the yellow and green luminescence may originate from the extrinsic emission due to the new energy states created by the oxygen-containing functional groups inside the band gap of CQDs. This novel strategy for fabricating optically tunable CQDs from coal is highly promising for the high-end application of coal.

\section{DATA AVAILABILITY STATEMENT}

The datasets generated for this study are available on request to the corresponding author.

\section{AUTHOR CONTRIBUTIONS}

QL, CZ, and JJ conceived and designed the experiments. YS, JC, YZ, and GH fabricated and characterized the sample. BX, LZ, GH, and YC analyzed the data. All authors discussed the experiment results and contributed to the writing of the paper.

\section{FUNDING}

This work was funded by the National Natural Science Foundation of China (21646006, U1704255, U1704146, $21805071,41472127)$ and the Natural Science Foundation of Henan province (162300410115).

Hoang, V. C., Hassan, M., and Gomes, V. G. (2018). Coal derived carbon nanomaterials-recent advances in synthesis and applications. Appl. Mater. Today 12, 342-358. doi: 10.1016/j.apmt.2018.06.007

Hu, C., Yu, C., Li, M., Wang, X., Yang, J., Zhao, Z., et al. (2015). Chemically tailoring coal to fluorescent carbon dots with tuned size and their capacity for Cu(II) detection. Small 10, 4926-4933. doi: 10.1002/smll.201401328

Hu, S., Wei, Z., Chang, Q., Trinchi, A., and Yang, J. (2016). A facile and green method towards coal-based fluorescent carbon dots with photocatalytic activity. Appl. Surf. Sci. 378, 402-407. doi: 10.1016/j.apsusc.2016.04.038

Huang, G., Geng, Q., Xing, B., Liu, Y., Li, Y., Liu, Q., et al. (2020). Manganous nitrate -assisted potassium hydroxide activation of humic acid to prepare oxygen-rich hierarchical porous carbon as high-performance supercapacitor electrodes. J. Power Sources 449:227506. doi: 10.1016/j.jpowsour.2019.227506

Jaleel, J. A., and Pramod, K. (2018). Artful and multifaceted applications of carbon dot in biomedicine. J. Control. Release 269, 302-321. doi: 10.1016/j.jconrel.2017.11.027

Kaur, M., Kaur, M., and Sharma, V. K. (2018). Nitrogen-doped graphene and graphene quantum dots: a review on synthesis and applications in energy, sensors and environment. Adv. Colloid Interfac. 259, 44-64. doi: 10.1016/j.cis.2018.07.001

Li, M., Hu, C., Yu, C., Wang, S., Zhang, P., and Qiu, J. (2015). Organic amine-grafted carbon quantum dots with tailored surface and enhanced photoluminescence properties. Carbon 91, 291-297. doi: 10.1016/j.carbon.2015.04.083

Li, M. Y., Yu, C., Hu, C., Yang, W. B., Zhao, C. T., Wang, S., et al. (2017) Solvothermal conversion of coal into nitrogen-doped carbon dots with singlet oxygen generation and high quantum yield. Chem. Eng. J. 320, 570-575. doi: $10.1016 /$ j.cej.2017.03.090 
Li, X., Rui, M., Song, J., Shen, Z., and Zeng, H. (2015). Carbon and graphene quantum dots for optoelectronic and energy devices: a review. Adv. Funct. Mater. 25, 4929-4947. doi: 10.1002/adfm.201501250

Li, Y., Liu, Y., Shang, X., Chao, D., Zhou, L., and Zhang, H. (2018). Highly sensitive and selective detection of $\mathrm{Fe}^{3+}$ by utilizing carbon quantum dots as fluorescent probes. Chem. Phys. Lett. 705, 1-6. doi: 10.1016/j.cplett.2018.05.048

Liu, F., Jang, M., Ha, H. D., Kim, J., Cho, Y., and Seo, T. S. (2013). Facile synthetic method for pristine graphene uantum dots and graphene oxide quantum dots: origin of blue and green luminescence. Adv. Mater. 25, 3657-3662. doi: 10.1002/adma.201300233

Liu, Q., Zhang, J., He, H., Huang, G., Xing, B., Jia, J., et al. (2018). Green preparation of high yield fluorescent graphene quantum dots from coal-tarpitch by mild oxidation. Nanomaterials 8, 844-853. doi: 10.3390/nano8100844

Liu, X., Hao, J., Liu, J., and Tao, H. (2018). Green synthesis of carbon quantum dots from lignite coal and the application in $\mathrm{Fe}^{3+}$ detection. IOP Conf. Seri. 113, 12063-12069. doi: 10.1088/1755-1315/113/1/0 12063

Liu, Y., Zhu, Y., Liu, S., Chen, S., Li, W., and Wang, Y. (2018). Molecular structure controls on micropore evolution in coal vitrinite during coalification. Int. J. Coal Geol. 199, 19-30. doi: 10.1016/j.coal.2018.09.012

Ma, Z., Yang, J., Zhang, J., Chen, G., Miao, C., Shi, L., et al. (2019). Layeropened graphene paper with carbon nanotubes as support in a flexible electrode material for supercapacitors. J. Alloy. Compd. 775, 982-989. doi: 10.1016/j.jallcom.2018.10.135

Moonrinta, S., Kwon, B., In, I., Kladsomboon, S., Sajomsang, W., and Paoprasert, P. (2018). Highly biocompatible yogurt-derived carbon dots as multipurpose sensors for detection of formic acid vapor and metal ions. Opt. Mater. 81, 93-101. doi: 10.1016/j.optmat.2018.05.021

Niekerk, D. V., Halleck, P. M., and Mathews, J. P. (2010). Solvent swelling behavior of Permian-aged South African vitrinite-rich and inertinite-rich coals. Fuel 89, 19-25. doi: 10.1016/j.fuel.2009.06.028

Okolo, G. N., Neomagus, H. W. J. P., Everson, R. C., Roberts, M. J., Bunt, J. R., Sakurovs, R., et al. (2015). Chemical-structural properties of South African bituminous coals: insights from wide angle XRD-carbon fraction analysis, ATR-FTIR, solid state ${ }^{13} \mathrm{C}$ NMR, and HRTEM techniques. Fuel 158, 779-792. doi: 10.1016/j.fuel.2015.06.027

Omer, K. M., Tofiq, D. I., and Ghafoor, D. D. (2019). Highly photoluminescent label free probe for Chromium (II) ions using carbon quantum dots co-doped with nitrogen and phosphorous. J. Lumin. 206, 540-546. doi: 10.1016/j.jlumin.2018.10.100

Pacquiao, M. R., de Luna, M. D. G., Thongsai, N., Kladsomboon, S., and Paoprasert, P. (2018). Highly fluorescent carbon dots from enokitake mushroom as multi-faceted optical nanomaterials for $\mathrm{Cr}^{6+}$ and VOC detection and imaging applications. Appl. Surf. Sci. 453, 192-203. doi: 10.1016/j.apsusc.2018.04.199

Pan, D., Zhang, J., Li, Z., and Wu, M. (2010). Hydrothermal route for cutting graphene sheets into blue-luminescent graphene quantum dots. Adv. Mater. 22, 734-738. doi: 10.1002/adma.200902825

Peng, L., Xu, Z., Liu, Z., Wei, Y., Sun, H., Li, Z., et al. (2015). An iron-based green approach to 1-h production of single-layer graphene oxide. Nat. Commun. 6, 5716-5725. doi: 10.1038/ncomms6716

Pramanik, A., Biswas, S., and Kumbhakar, P. (2018). Solvatochromism in highly luminescent environmental friendly carbon quantum dots for sensing applications: conversion of bio-waste into bio-asset. Spectrochim. Acta Part A 191, 498-512. doi: 10.1016/j.saa.2017.10.054

Qiu, H., Ni, W., Zhang, H., Chen, K., and Yu, J. (2020). Fabrication and evaluation of a regenerable HFO-doped agricultural waste for enhanced adsorption affinity towards phosphate. Sci. Total Environ. 703:135493. doi: 10.1016/j.scitotenv.2019.135493

Riyanto, S. I., Bindumadhavan, K., Chang, P., and Doong, R. (2019). Boron doped graphene quantum structure and MoS2 nanohybrid as anode materials for highly reversible lithium storage. Front. Chem. 7:116. doi: $10.3389 /$ fchem.2019.00116

Roy, S., Das, T., Ming, Y., Chen, X., Yue, C. Y., and Hu, X. (2014). Specific functionalization and polymer grafting on multiwalled carbon nanotubes to fabricate advanced nylon 12 composites. J. Mater. Chem. A 2, 3961-3970. doi: $10.1039 / \mathrm{c} 3$ ta $14528 \mathrm{j}$
Saikia, B. K., Saikia, M., Hower, J. C., Das, T., and Dutta, T. (2019). Feasibility study of preparation of carbon quantum dots from Pennsylvania anthracite and Kentucky bituminous coals. Fuel 243, 433-440. doi: 10.1016/j.fuel.2019.01.151

Sasikala, S. P., Henry, L., Yesilbag Tonga, G., Huang, K., Das, R., Giroire, B., et al. (2016). High yield synthesis of aspect ratio controlled graphenic materials from anthracite coal in supercritical fluids. ACS Nano 10, 5293-5303. doi: 10.1021/acsnano.6b01298

Shao, X., Wu, W., Wang, R., Zhang, J., Li, Z., Wang, Y., et al. (2016). Engineering surface structure of petroleum-coke-derived carbon dots to enhance electron transfer for photooxidation. J. Catal. 344, 236-241. doi: 10.1016/j.jcat.2016.09.006

Shi, W., Fan, H., Ai, S., and Zhu, L. (2015). Preparation of fluorescent graphene quantum dots from humic acid for bioimaging application. N. J. Chem. 39, 7054-7059. doi: 10.1039/C5NJ00760G

Syed, N., Huang, J., Feng, Y., Wang, X., and Cao, L. (2019). Carbon-based nanomaterials via heterojunction serving as photocatalyst. Front. Chem. 7:713. doi: $10.3389 /$ fchem. 2019.00713

Thiyagarajan, S. K., Raghupathy, S., Palanivel, D., Raji, K., and Raghupathy, P. (2016). Fluorescent carbon nano dots from lignite: unveiling the impeccable evidence for quantum confinement. Phys. Chem. Chem. Phys. 18, 12062-12073. doi: $10.1039 /$ С6CP00867D

Tian, Z., Zhang, X., Li, D., Zhou, D., Jing, P., Shen, D., et al. (2017). Full-color inorganic carbon dot phosphors for white-light-emitting diodes. Adv. Opt. Mater. 5, 1700416-1700424. doi: 10.1002/adom.201700416

Wang, F., Lu, Y., Chen, Y., Sun, J., and Liu, Y. (2018). Colorimetric nanosensor based on the aggregation of AuNP triggered by carbon quantum dots for detection of $\mathrm{Ag}^{+}$ions. ACS Sustain. Chem. Eng. 6, 3706-3713. doi: 10.1021/acssuschemeng.7b04067

Wang, X., Feng, Y., Dong, P., and Huang, J. (2019). A mini review on carbon quantum dots: preparation, properties, and electrocatalytic application. Front. Chem. 7:671. doi: 10.3389/fchem.2019.00671

Wu, M., Wang, Y., Wu, W., Hu, C., Wang, X., Zheng, J., et al. (2014). Preparation of functionalized water-soluble photoluminescent carbon quantum dots from petroleum coke. Carbon 78, 480-489. doi: 10.1016/j.carbon.2014. 07.029

Xing, B., Yuan, R., Zhang, C., Huang, G., Guo, H., Chen, Z., et al. (2017). Facile synthesis of graphene nanosheets from humic acid for supercapacitors. Fuel Process. Technol. 165, 112-122. doi: 10.1016/j.fuproc.2017. 05.021

Yan, Y., Chen, J., Li, N., Tian, J., Li, K., Jiang, J., et al. (2018). Systematic bandgap engineering of graphene quantum dots and applications for photocatalytic water splitting and $\mathrm{CO}_{2}$ reduction. ACS Nano 12, 3523-3532. doi: 10.1021/acsnano.8b00498

Yang, Q., Yang, W., Yang, P., Duan, J., Li, X., Mo, J., et al. (2018). Nitrogendoped carbon quantum dots from biomass via simple one-pot method and exploration of their application. Appl. Surf. Sci. 434, 1079-1085. doi: 10.1016/j.apsusc.2017.11.040

Ye, R., Peng, Z., Metzger, A., Lin, J., Mann, J. A., Huang, K., et al. (2015). Bandgap engineering of coal-derived graphene quantum dots. ACS Appl. Mater. Inter. 7, 7041-7048. doi: 10.1021/acsami.5b01419

Ye, R., Xiang, C., Lin, J., Peng, Z., Huang, K., Yan, Z., et al. (2013). Coal as an abundant source of graphene quantum dots. Nat. Commun. 4, 2943-2948. doi: $10.1038 /$ ncomms 3943

Yoon, H., Chang, Y. H., Song, S. H., Lee, E., Jin, S. H., Park, C., et al. (2016). Intrinsic photoluminescence emission from subdomained graphene quantum dots. Adv. Mater. 28, 5255-5261. doi: 10.1002/adma.201600616

Yu, X., Liu, J., Yu, Y., Zuo, S., and Li, B. (2014). Preparation and visible light photocatalytic activity of carbon quantum dots/ $\mathrm{TiO}_{2}$ nanosheet composites. Carbon 68, 718-724. doi: 10.1016/j.carbon.2013.11.053

Zeng, H., Xing, B., Zhang, C., Chen, L., Zhao, H., Han, X., et al. (2020). In situ synthesis of $\mathrm{MnO}_{2}$ /porous graphitic carbon composites as highcapacity anode materials for lithium-ion batteries. Energ. Fuel. 34, 2480-2491. doi: 10.1021/acs.energyfuels.9b04325

Zhang, B., Maimaiti, H., Zhang, D., Xu, B., and Wei, M. (2017). Preparation of coal-based C-dots/ $/ \mathrm{TiO}_{2}$ and its visible-light photocatalytic characteristics for degradation of pulping black liquor. J. Photochem. Photobiol. 345, 54-62. doi: 10.1016/j.jphotochem.2017.05.031 
Zhang, F., Liu, F., Wang, C., Xin, X., Liu, J., Guo, S., et al. (2016). Effect of lateral size of graphene quantum dots on their properties and application. ACS Appl. Mater. Inter. 8, 2104-2110. doi: 10.1021/acsami.5b10602

Zhang, L., Wang, Z., Zhang, J., Jia, J., Zhao, D., and Fan, Y. (2018). Phenanthrolinederivative functionalized carbon dots for highly selective and sensitive detection of $\mathrm{Cu}^{2+}$ and $\mathrm{S}^{2--}$ and imaging inside live cells. Nanomaterials 8 , 1071-1081. doi: 10.3390/nano8121071

Zhang, Q., Bolisetty, S., Cao, Y., Handschin, S., Adamcik, J., Peng, Q., et al. (2019). Selective and efficient removal of fluoride from water by in-situ engineered amyloid fibrils- $\mathrm{ZrO}_{2}$ hybrid membranes. Angew. Chem. Int. Edn. 58, 6012-6016. doi: 10.1002/anie.201901596

Zhang, Q., Yang, Q., Phanlavong, P., Li, Y., Wang, Z., Jiao, T., et al. (2017). Highly efficient lead(II) sequestration using size-controllable polydopamine microspheres with superior application capability and rapid capture. ACS Sustain. Chem. Eng. 5, 4161-4170. doi: 10.1021/acssuschemeng.7b 00129

Zhang, Z., and Xu, X. (2015). Nondestructive covalent functionalization of carbon nanotubes by selective oxidation of the original defects with $\mathrm{K}_{2} \mathrm{FeO}_{4}$. Appl. Surf. Sci. 346, 520-527. doi: 10.1016/j.apsusc.2015.04.026
Zhou, L., Qiao, M., Zhang, L., Sun, L., Zhang, Y., and Liu, W. (2019). Green and efficient synthesis of carbon quantum dots and their luminescent properties. $J$. Lumin. 206, 158-163. doi: 10.1016/j.jlumin.2018.10.057

Zhu, S., Meng, Q., Wang, L., Zhang, J., Song, Y., Jin, H., et al. (2013). Highly photoluminescent carbon dots for multicolor patterning, sensors, and bioimaging. Angew. Chem. Int. Edn. 52, 3953-3957. doi: 10.1002/anie.201300519

Conflict of Interest: The authors declare that the research was conducted in the absence of any commercial or financial relationships that could be construed as a potential conflict of interest.

Copyright (C) 2020 Jia, Sun, Zhang, Liu, Cao, Huang, Xing, Zhang, Zhang and Cao. This is an open-access article distributed under the terms of the Creative Commons Attribution License (CC BY). The use, distribution or reproduction in other forums is permitted, provided the original author(s) and the copyright owner(s) are credited and that the original publication in this journal is cited, in accordance with accepted academic practice. No use, distribution or reproduction is permitted which does not comply with these terms. 